\title{
de Sitter symmetries and inflationary scalar-vector models
}

\author{
Josue Motoa-Manzano ${ }^{1, *}$, Juan P. Beltrán Almeida ${ }^{2, * *}$, César A. Valenzuela-Toledo ${ }^{1, * * *}$
}

Edited by

Juan Carlos Salcedo-Reyes

(salcedo.juan@javeriana.edu.co)

1. Departamento de Física, Universidad del Valle, Ciudad Universitaria Meléndez, Santiago de Cali 760032, Colombia.

2. Departamento de Física, Universidad Antonio Nariño, Cra 3 Este \# 47A-15,

Bogotá D.C. 110231, Colombia.

*josue.motoa@correounivalle.edu.co

**juanpbeltran@uan.edu.co

***cesar.valenzuela@correounivalle.edu.co

Received: 01-05-2016

Accepted: 08-09-2016

Published on line: 12-10-2016

Citation: Motoa-Manzano J, Beltrán

Almeida JP, Valenzuela-Toledo CA.

de Sitter symmetries and inflationary

scalar-vector models,

Universitas Scientiarum, 21 (3): 219-243, 2016.

doi: 10.11144/Javeriana.SC21-3.dssa

\section{Funding:}

COLCIENCIAS grant numbers 110656933958 RC 0384-2013 and 123365843539 RC FP44842-081-2014, COLCIENCIAS-ECOS NORD grant number RC 0899-2012 and Vicerrectoría de Investigaciones - Univalle grant number 7924

Electronic supplementary material: $\mathrm{N} / \mathrm{A}$

OPEN ACCESS

\section{Abstract}

In this paper, we study the correspondence between a field theory in de Sitter space in $D$-dimensions and a dual conformal field theory in a euclidean space in $(D-1)$-dimensions. In particular, we investigate the form in which this correspondence is established for a system of interacting scalar and a vector fields propagating in de Sitter space. We analyze some necessary (but not sufficient) conditions for which conformal symmetry is preserved in the dual theory in $(D-1)$-dimensions, making possible the establishment of the correspondence. The discussion that we address in this paper is framed on the context of inflationary cosmology. Thusly, the results obtained here pose some relevant possibilities of application to the calculation of the fields's correlation functions and of the primordial curvature perturbation $\zeta$, in inflationary models including coupled scalar and vector fields.

Keywords: Inflation; vector fields; de Sitter symmetries; parity violation.

\section{Introduction}

The study of the symmetries is very important when we look for fundamental descriptive features of a particular physical system and solve its dynamics. For example, it is well known that through the Noether theorem we can identify conserved quantities related to the symmetries, which allows us to discover intrinsic fundamental characteristics that will be reflected on the dynamics of the system. It is also well known that the geometry of the inflationary period of our universe is described with great accuracy by the de Sitter space.

This spacetime is similar to the Minkowski spacetime in the sense that it is a maximally symmetric space, which means that in $D$ dimensions, the number of symmetry generators is $D(D+1) / 2$. This fact defines and restricts the 
form of the kinematic features and the evolution of a system on this space. During the inflationary period, the symmetries of the de Sitter group play an essential role on the description of the primordial perturbations and their statistical properties.

In particular, by using the correspondence between the de Sitter symmetry group in $D$-dimensions and the conformal group in an euclidean $(D-1)$ dimensional "boundary" space (Strominger, 2001), it is possible to calculate general important features of the system, such as the correlations functions of the fields that govern the dynamics of the inflationary Universe (Biagetti et al., 2013), which are necessary to calculate the statistical descriptors of the primordial curvature perturbation by using, for example, the $\delta \mathrm{N}$ formalism (Sasaki \& Stewart, 1996; Nakamura \& Stewart, 1996; Starobinsky, 1985; Lyth et al., 2005; Dimopoulos et al., 2009) or cosmological perturbation theory (Gumrukcuoglu et al., 2010; Bartolo et al., 2013). To do that, we need first to calculate one of the fundamental quantities of the conformal field theory description: the conformal weights of the fields.

The correspondence between a field theory in de Sitter space and a dual theory in a euclidean boundary space (one dimension less than the de Sitter space) is what we shall refer to as $\mathrm{dS} / \mathrm{CFT}$ correspondence and it is at the core of the approach that we are pursuing here. This correspondence was proposed initially for free non interacting single fields propagating on de Sitter space (Bousso et al., 2002) but some considerations about the interacting case was discussed in the literature (Spradlin \& Volovich, 2002)

In this paper, we follow the approach proposed by (Biagetti et al., 2013) to study the case of a coupled scalar-vector system. In the context that we are framing our discussion, the scalar field is responsible for the inflationary expansion and the vector field is partly responsible of the generation of the primordial curvature perturbation $\zeta$. The inclusion of vector fields on the inflationary dynamics permits the study of several interesting possibilities such as the presence of statistical anisotropies, parity violating patterns, the origin of primordial magnetic fields, etc. (see for instance Caprini \& Sorbo, 2014; Maleknejad et al., 2013; Soda, 2012; Dimastrogiovanni et al., 2010, and references therein). For concreteness, we will settle the discussion in four dimensional de Sitter space but the results obtained can be generalised straightforwardly to any dimensions.

This paper is organized as follows. In section II, we review the symmetries and some basics of de Sitter space. Next, in sections III and IV, we shall describe the transformation laws imposed by these symmetries over the conformal 
fields on the dual theory and we calculate the associated conformal weights of those fields. In section $\mathrm{V}$, we briefly review the results for the scalar and the vector field separately and then, in section VI, we consider the coupled scalar-vector system. Finally, in section VII, we present our conclusions and discuss some possible applications of the results obtained.

\section{Symmetries of the de Sitter Space}

The de Sitter spacetime can be described in several useful coordinate systems (for reviews see for instance Spradlin et al., 2001 and Leblond et al., 2002), but for our interests, we will chose the $4 \mathrm{D}$ conformal planar coordinates in which the line element is written as

$$
d s^{2}=\frac{1}{(H \tau)^{2}}\left(-d \tau^{2}+d \vec{x}^{2}\right),
$$

where $H$ is the Hubble parameter and $\tau$ is the conformal time. In this coordinate system, it is easy to see that the line element (1) is invariant under spacial translations and rotations on $\tau=$ constant sections:

$$
x_{i}^{\prime}=a_{i}+R_{i j} x_{j}
$$

where $a_{i}$ is a 3-dimensional constant vector and $R_{i j}$ is an $O(3)$ matrix satisfying the condition $R_{i k} R^{k j}=\delta_{i}^{j}$ and representing a three dimensional rotation. We have a total of six symmetry generators included in the transformation (2) and they affect only the spacial sections of the spacetime.

Also, notice that we allow the possibility of spacial reflexion $x^{i} \rightarrow-x^{i}$ in this group of transformations. Spacial reflexion is an explicit symmetry of the line element (1) and we will pay attention to this specific transformation, when we consider the possibility of introducing parity violating models in the presence of vector fields. There are two additional transformation which mixes the time and the spacial coordinates, the spacetime dilatation:

$$
x^{\prime \mu}=\lambda x^{\mu} \rightarrow \quad x_{i}^{\prime}=\lambda x_{i}, \quad \tau \rightarrow \tau^{\prime}=\lambda \tau,
$$

where $\lambda$ is a constant factor and the special conformal transformation:

$$
x^{\prime \mu}=\frac{x^{\mu}+b^{\mu} x^{2}}{1+2 \vec{b} \cdot \vec{x}+b^{2} x^{2}},
$$

or

$$
x_{i}^{\prime}=\frac{x_{i}+b_{i}\left(-\tau^{2}+\vec{x}^{2}\right)}{1+2 \vec{b} \cdot \vec{x}+b^{2}\left(-\tau^{2}+\vec{x}^{2}\right)},
$$

and 


$$
\tau^{\prime}=\frac{\tau}{1+2 \vec{b} \cdot \vec{x}+b^{2}\left(-\tau^{2}+\vec{x}^{2}\right)},
$$

with $b^{\mu}=\left(0, b_{i}\right)$ a three dimensional vector which generates the transformation.

In the last expressions we use the notation $x^{2}=\eta_{\mu \nu} x^{\mu} x^{\nu}, \eta_{\mu \nu}=\operatorname{diag}(-1,1,1$, 1), $\overrightarrow{x^{2}}=\delta_{i j} x^{i} x^{j}$ and $\vec{x} \cdot \vec{b}=\delta_{i j} b^{i} x^{j}$. Together, the transformations (2), (3) and (4) tell us that de Sitter space has ten symmetry generators. This is a maximally symmetric spacetime. It can be seen that (4) is constructed out by the composition of three consecutive transformations, namely, an inversion, written as

$$
x^{\prime \mu}=\frac{x^{\mu}}{x^{2}}
$$

or

$$
x_{i}^{\prime}=\frac{x_{i}}{-\tau^{2}+\vec{x}^{2}}, \quad \tau^{\prime}=\frac{\tau}{-\tau^{2}+\vec{x}^{2}},
$$

then a translation $\left(\tau^{\prime} \rightarrow \tau^{\prime \prime}=\tau^{\prime}\right.$ and $\left.x_{i}^{\prime} \rightarrow x_{i}^{\prime \prime}=x_{i}^{\prime}+b_{i}\right)$ and then an inversion again. Actually, the special conformal transformation (4) can be expressed as:

$$
\frac{x^{\prime \mu}}{x^{\prime 2}}=\frac{x^{\mu}}{x^{2}}+b^{\mu}
$$

It becomes useful in the following to have the Jacobian matrices associated to the coordinate transformations of the de Sitter isometries. Particularly, we will need the Jacobian for the dilatation and for special conformal transformation (3) and (4). Nevertheless, as we said before, the special conformal transformation can be obtained by composing translations and inversions, allowing us to use the transformation (6) instead of (4).

The Jacobian matrix of the inversion, can be derived directly for (6) obtaining

$$
\frac{\partial x^{\prime \mu}}{\partial x^{\nu}}=\frac{1}{x^{2}}\left(\delta_{\mu}^{\nu}-2 \frac{x^{\mu} \eta_{\nu \alpha} x^{\alpha}}{x^{2}}\right)=\frac{1}{x^{2}} J_{\nu}^{\mu},
$$

where $J_{\mu}^{\nu}$ satisfies the orthogonality relation $J_{\mu}^{\sigma} J_{\sigma}^{\nu}=\delta_{\mu}^{\nu}$. The associated Jacobian determinant of the above transformation is

$$
\operatorname{det}\left(\frac{\partial x^{\prime \mu}}{\partial x^{\nu}}\right)=-\frac{1}{\left(x^{2}\right)^{4}}
$$

Using (6), we can also calculate the Jacobian of the inverse transformation $x^{\prime} \rightarrow x$, obtaining 


$$
\frac{\partial x^{\mu}}{\partial x^{\prime \nu}}=x^{2} J_{\nu}^{\mu}, \quad \operatorname{det}\left(\frac{\partial x^{\mu}}{\partial x^{\prime \nu}}\right)=\left(x^{2}\right)^{4} .
$$

On the other hand, for the dilatations we have:

$$
\begin{array}{rlrl}
x^{\prime \mu} & =\lambda x^{\mu}, \\
\frac{\partial x^{\prime \mu}}{\partial x^{\nu}} & =\lambda \delta_{\nu}^{\mu}, & \operatorname{det}\left(\frac{\partial x^{\prime \mu}}{\partial x^{\nu}}\right)=\lambda^{4}, \\
\frac{\partial x^{\mu}}{\partial x^{\prime \nu}} & =\frac{1}{\lambda} \delta_{\nu}^{\mu}, & \operatorname{det}\left(\frac{\partial x^{\mu}}{\partial x^{\prime \nu}}\right)=\lambda^{-4} .
\end{array}
$$

Then, with the previous results, we can also calculate the change in the volume element, which reads:

$$
d \tau^{\prime} d^{3} x^{\prime}=\left|\operatorname{det}\left(\frac{\partial x^{\prime \mu}}{\partial x^{\nu}}\right)\right| d \tau d^{3} x .
$$

In next sections, we will exploit the correspondence between the de Sitter isometry group represented by the transformations below and the conformal group in $\mathbb{R}^{3}$. In the essence of this correspondence lies the idea that de Sitter isometries act as the conformal group transformations in $\mathbb{R}^{3}$, a fact that we will show on next section. Particularly, we will consider the asymptotic region, where inflationary perturbations are frozen, after horizon crossing at supper Hubble scales which in these coordinates happens for $-\tau \ll|\vec{x}|$. Then, in the following section, we will recall some basis of the conformal group and introduce the relevant terminology that will allow us to construct a conformal field theory representation of the fields in de Sitter space.

An important part of the following discussion is devoted to the conformal weight of the fields in the conformal field theory representation. The conformal weight of the field is a crucial element for expressing the transformation rules of the fields and the symmetries of their correlation functions which are the meaningful objects constructed in the theory because they encode the statistical properties of the theory.

\section{Conformal group basics and relation with de Sitter group symmetries}

In an Euclidean space, the conformal group is defined as the set of coordinate transformations $x \rightarrow x^{\prime}$ that leave invariant the angles between two vectors in this space, or, equivalently, the group of transformations that leave invariant the metric up to a factor

$$
g_{\mu \nu}(x) \rightarrow g_{\mu \nu}^{\prime}\left(x^{\prime}\right)=\omega(x) g_{\mu \nu}(x)
$$


where $\omega(x)$ is an arbitrary function of the coordinates. It is easy to see that the transformations (2), (3) and (4) act as conformal transformations on $\mathbb{R}^{3}$ for the euclidean metric, i.e. $g_{i j}=\delta_{i j}$, in (15) with the corresponding conformal factor $\omega$ :

$$
\begin{aligned}
& x_{i}^{\prime}=a_{i}+R_{i j} x_{j} \quad \rightarrow \quad \omega=1, \\
& x_{i}^{\prime}=\lambda x_{i} \quad \rightarrow \quad \omega=\lambda^{-2} \\
& x_{i}^{\prime}=\frac{x_{i}+b_{i} \vec{x}^{2}}{1+2 \vec{b} \cdot \vec{x}+b^{2}\left(\vec{x}^{2}\right)} \rightarrow \omega=\left(1+2 \vec{b} \cdot \vec{x}+b^{2} \vec{x}^{2}\right)^{2} .
\end{aligned}
$$

Then, we can see explicitly that the de Sitter symmetry group in four dimensions induces a conformal group in the three dimensional space. In few words, we can interpret the symmetries (16) to (18) as the asymptotic symmetry group of the boundary region of the four dimensional de Sitter space time. In our context, the asymptotic region is located at super horizon scales when the inflationary perturbations evolve classically and carry the information of the physical mechanism that drives the inflationary expansion. In the coordinates employed here, and in the previous expressions, we have used that the super horizon limit which constitutes our asymptotic euclidean space lies in the region $|\tau| \ll|\vec{x}|$. It its worth to notice that we obtain ten symmetry group generators as we expect for the conformal group acting on three dimensional space.

Additionally, or equivalently to the expression (18) we can write the inversion transformation and its associated conformal factor as:

$$
x_{i}^{\prime}=\frac{x_{i}}{\left(\vec{x}^{2}\right)} \rightarrow \omega=\left(\vec{x}^{2}\right)^{2} .
$$

So far, we were talking about the symmetries and the geometry of the spacetime, and now we turn to the fields on the theory. The relevant objects that we need for the conformal field theory description, are the ones that we shall call a primary field. A primary field is an object which transforms according to the following rule under conformal transformations

$$
\begin{aligned}
T_{i_{1} \ldots i_{n}}(x) \rightarrow T_{i_{1} \ldots i_{n}}^{\prime}\left(x^{\prime}\right)= & \left|\operatorname{det}\left(\frac{\partial x^{l}}{\partial x^{k}}\right)\right|^{\frac{n-\Delta_{T}}{d}} \times \\
& \frac{\partial x^{j_{1}}}{\partial x^{\prime i_{1}}} \ldots \frac{\partial x^{j_{n}}}{\partial x^{\prime i_{n}}} T_{j_{1} \ldots j_{n}}(x),
\end{aligned}
$$

where $n$ is the order of the tensor, $d$ is the dimension of the spacetime and $\Delta_{T}$ is the conformal weight of the field (Biagetti et al., 2013). When dealing with the conformal dual theory to the theory in de Sitter space, we will consider the Euclidean space $\mathbb{R}^{3}$. 
By using the above transformation, we can find the conformal dimension $\Delta_{T}$ of different fields that are involved in the theory. It is beyond the scope of this short article to enter in the details of the conformal field theory machinery, so we will just restrict to the introduction of the fundamental elements necessary for our purpose. A complete study of conformal field theory can be found for instance in (Ginsparg, 1988)

\section{Fields on de Sitter space}

Now, we will study of fields propagating on de Sitter space. With the results obtained in the previous section, we can evaluate the conformal weight, which is an essential quantity necessary for the conformal field theory description of the fields. We are interested in the coupled scalar vector system, but, before that, and mainly for illustrative reasons and for developing the technique introducing the necessary elements, we study the single scalar field and the single vector field cases separately. After that and invoking the results from the single field case, we will face the coupled system.

Single scalar field. The action for a single massive scalar field propagating on de Sitter space in the coordinates (1) is given by

$$
\begin{aligned}
S_{\phi} & =-\frac{1}{2} \int d^{4} x \sqrt{-g}\left\{\partial_{\mu} \phi \partial^{\mu} \phi+m_{\phi}^{2} \phi^{2}\right\} \\
& =-\frac{1}{2} \int \frac{d \tau d^{3} x}{H^{2} \tau^{2}}\left\{\eta^{\mu \nu} \partial_{\mu} \phi \partial_{\nu} \phi+\frac{m_{\phi}^{2} \phi^{2}}{H^{2} \tau^{2}}\right\},
\end{aligned}
$$

where $m_{\phi}$ is the mass of the field. Now, we study the symmetries of the theory in order to learn about the transformation of the fields. To emphasise the difference between the properties of the scalar field in de Sitter space and in $\mathbb{R}^{3}$, let us suppose that we write the transformation law for a scalar in de Sitter as in (20):

$$
\phi^{\prime}=\left|\operatorname{det}\left(\frac{\partial x^{\prime \mu}}{\partial x^{\nu}}\right)\right|^{-\frac{\Delta_{\phi}}{4}} \phi
$$

and for the action

$$
S_{\phi}^{\prime}=-\frac{1}{2} \int d \tau^{\prime} d x^{\prime 3} \frac{1}{H^{2} \tau^{\prime 2}}\left\{\eta^{\mu \nu} \partial_{\mu}^{\prime} \phi^{\prime} \partial_{\nu}^{\prime} \phi^{\prime}+\frac{m_{\phi}^{2} \phi^{\prime 2}}{H^{2} \tau^{\prime 2}}\right\} .
$$

Under the inversion (6) we have: $\phi^{\prime}=\left(x^{2}\right)^{\Delta_{\phi}} \phi$ and 


$$
\begin{aligned}
S_{\phi}^{\prime}= & \int d \tau d x^{3} \frac{1}{H^{2} \tau^{2}}\left\{-4 \Delta_{\phi}^{2}\left(x^{2}\right)^{2 \Delta_{\phi}} \phi^{2}\right. \\
& -4 \Delta_{\phi}\left(x^{2}\right)^{2 \Delta_{\phi}-1} \phi x^{\mu} \partial_{\mu} \phi-\left(x^{2}\right)^{2 \Delta_{\phi}} \\
& \left.\times\left[\eta^{\mu \nu} \partial_{\mu} \phi \partial_{\nu} \phi+\frac{m_{\phi}^{2} \phi^{2}}{H^{2} \tau^{2}}\right]\right\},
\end{aligned}
$$

where we have used $\partial_{\mu}^{\prime}=x^{2} J_{\mu}^{\nu} \partial_{\nu}$.

Demanding invariance of the action $S_{\phi}=S_{\phi}^{\prime}$ we see that it is necessary to set $\Delta_{\phi}=0$, and then $\phi^{\prime}=\phi$. It is easy to arrive to the same result if we apply the dilatation (3) and it is trivial for the translations and three dimensional rotations (2) because the transformation matrix is orthogonal. Certainly, this result does not come as a surprise at all, since we are talking about of a scalar field. Nevertheless it is worth to stress this fact because we are going to see that asymptotically, in the super horizon limit the dual field associated to the scalar transform according with (20) with a conformal weight different than $\Delta_{\phi}=0$.

Now, we study the behaviour of the associated conformal field of the scalar filed in the three dimensional space $\mathbb{R}^{3}$. To this end, it is necessary to solve the equation of motion and go to super horizon scales. The equation of motion derived from (21) is

$$
\ddot{\phi}-\frac{2}{\tau} \dot{\phi}-\nabla^{2} \phi+\frac{m_{\phi}^{2} \phi}{H^{2} \tau^{2}}=0 .
$$

To solve this equation, we go to momentum space. Using the Fourier transform of the field, $F(\tau, \vec{x})=\int \frac{d^{3} x}{(2 \pi)^{3 / 2}} \tilde{F}(\tau, \vec{k}) e^{i \vec{k} \cdot \vec{x}}$, the resulting equation is:

$$
\ddot{\tilde{\phi}}-\frac{2}{\tau} \dot{\tilde{\phi}}+k^{2} \tilde{\phi}+\frac{m_{\phi}^{2} \tilde{\phi}}{H^{2} \tau^{2}}=0,
$$

which can be fully solved analytically in terms of Bessel functions $J_{\nu}$ and $Y_{\nu}$ :

$$
\tilde{\phi}(\tau, \vec{k})=\tau^{3 / 2}\left(C_{1}(\vec{k}) J_{h}(k \tau)+C_{2}(\vec{k}) Y_{h}(k \tau)\right),
$$

where $C_{1}$ and $C_{2}$ are constants which depends on boundary initial conditions and

$$
h=\sqrt{\frac{9}{4}-\frac{m_{\phi}^{2}}{H^{2}}} .
$$


Now, we need the behaviour of the solution (25) at super horizon scales, which in momentum space are achieved when $-k \tau \ll 1$ (Tanhayi, 2014). By expanding the Bessel functions in that regime and going back to $(\tau, \vec{x})$ space, we arrive to

$$
\phi(\tau, \vec{x}) \approx \tau^{\frac{3}{2}+h} \sigma_{+}(\vec{x})+\tau^{\frac{3}{2}-h} \sigma_{-}(\vec{x})
$$

where we have separated explicitly the time depending part. The precise interpretation of the previous result depends on the value of the discriminant (26) because depending if $m_{\phi}<\frac{3}{2} H$ or $m_{\phi}>\frac{3}{2} H$. We can provide a unitary interpretation of the conformal field theory associated, we don't enter into this discussion here which is beyond the scope of this paper and address the attention of the reader to Strominger (2001), Bousso et al. (2002) and Spradlin $\&$ Volovich (2002). In the following, we restrict ourselves to the case $m_{\phi}<\frac{3}{2} H$. The dominant solution for super horizon regime in this case is $\tau^{\frac{3}{2}-h}$, so we have that

$$
\lim _{|\tau| \rightarrow 0} \phi(\tau, \vec{x})=\tau^{\frac{3}{2}-h} \sigma_{-}(\vec{x}) .
$$

The field $\sigma$ is the one that we need to construct the dual conformal theory for late times after horizon crossing, so, we need to determine if it behave as a conformal field by deriving its transformation under conformal transformations in $\mathbb{R}^{3}$, actually, as we comment before, we just need the transformation for dilatations (3) and inversions (6) in 3D. Before that, we need to take the super horizon limit $|\tau| \ll|\vec{x}|$ of the inversion (6): $\tau^{\prime}=\frac{\tau}{|\vec{x}|^{2}}, x_{i}^{\prime}=\frac{x_{i}}{|\vec{x}|^{2}}$. Using $\phi^{\prime}=\phi$, and (3) we obtain

$$
\begin{aligned}
\phi^{\prime}\left(\tau^{\prime}, x_{i}^{\prime}\right) & =\tau^{\prime \frac{3}{2}-h} \sigma^{\prime}\left(\vec{x}^{\prime}\right)=\lambda^{\frac{3}{2}-h} \tau^{\frac{3}{2}-h} \sigma^{\prime}\left(\vec{x}^{\prime}\right) \\
& =\tau^{\frac{3}{2}-h} \sigma(\vec{x})=\phi\left(\tau, x_{i}\right)
\end{aligned}
$$

which implies:

$$
\sigma^{\prime}\left(\vec{x}^{\prime}\right)=\lambda^{-\left(\frac{3}{2}-h\right)} \sigma(\vec{x})
$$

On the other hand, for inversions in the super horizon regime

$$
\begin{array}{r}
\phi^{\prime}\left(\tau^{\prime}, x_{i}^{\prime}\right)=\tau^{\prime \frac{3}{2}-h} \sigma^{\prime}\left(\vec{x}^{\prime}\right)=\frac{\tau^{\frac{3}{2}-h}}{|\vec{x}|^{2\left(\frac{3}{2}-h\right)}} \sigma^{\prime}\left(\vec{x}^{\prime}\right) \\
=\tau^{\frac{3}{2}-h} \sigma(\vec{x})=\phi\left(\tau, x_{i}\right),
\end{array}
$$

which implies

$$
\sigma^{\prime}\left(\vec{x}^{\prime}\right)=|\vec{x}|^{2\left(\frac{3}{2}-h\right)} \sigma(\vec{x})
$$


When we compare (30) and (32) with (20) (with $n=0$ and $d=3$ ) we conclude that the field $\sigma$ behaves as a conformal field with conformal weight

$$
\Delta_{\sigma}=\frac{3}{2}-h=\frac{3}{2}-\sqrt{\frac{9}{4}-\frac{m_{\phi}^{2}}{H^{2}}} .
$$

This is an important result given that the correlating functions of a conformal field theory can be calculated if we know the conformal weight of the primary fields. In this case, we expect that the 2 point correlating function of the dual theory of a scalar field on de Sitter space behave like

$$
\left\langle\mathcal{O}_{\sigma}(\vec{x}) \mathcal{O}_{\sigma}(\vec{y})\right\rangle=\frac{A}{|\vec{x}-\vec{y}|^{\Delta_{\sigma}}}
$$

with $A$ a constant. For an detailed analysis of the two point correlation functions in the dual theory see e.g. Strominger (2001) and Leblond et al. (2003).

Single vector field. Now, we turn to the vector field case. We consider a massive vector field with Lagrangian

$$
S_{A}=\int d \tau d^{3} x \sqrt{-g}\left\{-\frac{1}{4} F_{\mu \nu} F^{\mu \nu}-m_{A}^{2} A_{\mu} A^{\mu}\right\},
$$

where $F_{\mu \nu}=\partial_{\mu} A_{\nu}-\partial_{\nu} A_{\mu}$ and $m_{A}$ is the mass of the vector field. This model have been studied with some detail in Ackerman et al. (2007) and (allowing for non-minimal coupling) Golovnev et al. (2008) and in that time, it sparked some interest in the study of inflationary models with vector fields. In de Sitter conformal coordinates (1) we have the action

$$
\begin{array}{r}
S_{A}=\int d \tau d^{3} x\left\{-\frac{1}{2} \eta^{\mu \alpha} \eta^{\beta \nu} \partial_{\nu} A_{\mu}\left(\partial_{\beta} A_{\alpha}-\partial_{\alpha} A_{\beta}\right)\right. \\
\left.-\frac{m_{A}^{2} \eta^{\mu \nu} A_{\mu} A_{\nu}}{H^{2} \tau^{2}}\right\} .
\end{array}
$$

Again, as we did in the scalar case, we will consider that $A_{\mu}$ transforms as in (20) in the following way

$$
A_{\mu}^{\prime}=\left(x^{2}\right)^{\Delta_{A}} J_{\mu}^{\nu} A_{\nu}
$$

and using inversion we get the transformation of the action with respect to the change of coordinates 


$$
\begin{array}{r}
S_{A}^{\prime}=\int d \tau d^{3} x \frac{\left(x^{2}\right)^{2+2 \Delta_{A}}}{\left(x^{2}\right)^{4}}\left\{-2 \frac{\left(\Delta_{A}-1\right)^{2}}{\left(x^{2}\right)^{2}} A_{\mu} A_{\nu}\right. \\
\times\left(x^{2} \eta^{\mu \nu}-x^{\mu} x^{\nu}\right)-2 \frac{\left(\Delta_{A}-1\right)}{\left(x^{2}\right)^{2}} x^{\mu} A_{\alpha} \eta^{\alpha \nu} \times \\
\left(\partial_{\mu} A_{\nu}-\partial_{\nu} A_{\mu}\right)-\frac{1}{2} \eta^{\mu \alpha} \eta^{\nu \beta} \partial_{\nu} A_{\mu}\left(\partial_{\alpha} A_{\beta}-\partial_{\beta} A_{\alpha}\right) \\
\left.-\frac{m_{A}^{2} \eta^{\mu \nu} A_{\mu} A_{\nu}}{H^{2} \tau^{2}}\right\},
\end{array}
$$

from which we deduce that invariance under inversions implies that $\Delta_{A}=1$. Again, this is not surprising since this is precisely the transformation law for a vector in four dimensional de Sitter space.

Now, we look if there is an associated three dimensional vector which transforms as a conformal vector field in $\mathbb{R}^{3}$. We begin by solving the equations of motion derived from the action (36)

$$
\eta^{\beta \lambda} \partial_{\lambda}\left(\partial_{\beta} A_{\nu}-\partial_{\nu} A_{\beta}\right)-\frac{m_{A}^{2} A_{\nu}}{(H \tau)^{2}}=0 .
$$

The system before propagate three degrees of freedom due to the presence of the mass term, two transverse terms perpendicular to the propagation axis, and a longitudinal term for the equation of the component $A_{0}$. It is well known that the longitudinal mode in this model is problematic due to the presence of ghost instabilities (Himmetoglu et al., (2009a); Himmetoglu et al., (2009b)), but it is also known that this problem can be solved by introducing a time dependent coupling in the Maxwell term $f(\tau) F^{2}$ and a time dependent mass term $m(\tau)$ (Dimopoulos et al., (2010a); Dimopoulos et al., 2010b)).

We avoid the analysis of the longitudinal mode which is not relevant for our main objectives here, we refer the reader to the references mentioned before and in the following we just focus on the transverse components that can be obtained from the divergence free spacial components $\left(\partial_{i} A_{i}=0\right)^{1}$ :

$$
\ddot{A}_{i}-\delta^{k l} \partial_{k} \partial_{l} A_{i}+\frac{m_{A}^{2} A_{i}}{(H \tau)^{2}}=0 .
$$

Again, in a similar way that in the scalar case, we solve the equation for the transverse component in momentum space

$$
\tilde{A}_{i}(\tau, \vec{k})=\sqrt{\tau}\left(D_{1} J_{p}(k \tau)+D_{2} Y_{p}(k \tau)\right) \tilde{T}_{i}(\vec{k}),
$$

where $\tilde{A}_{i}$ is the Fourier transform of the vector field, and

\footnotetext{
${ }^{1}$ In general, we always can separate a vector field in a transverse and a longitudinal part: $A_{\mu}=A_{\mu}^{\perp}+A_{\mu}^{\|}$with $\partial^{\mu} A_{\mu}^{\perp}=0$.
} 


$$
p=\sqrt{\frac{1}{4}-\frac{m_{A}^{2}}{H^{2}}}
$$

Going to super horizon scales at $|k \tau| \ll 1$ and going back to coordinates space we get the asymptotic expansion

$$
A_{i}(\tau, \vec{x}) \approx \tau^{\frac{1}{2}+p} V_{i}(\vec{x})+\tau^{\frac{1}{2}-p} U_{i}(\vec{x}) .
$$

For late times, again we have that the dominant term is $\tau^{\frac{1}{2}-p}$, then:

$$
\lim _{|\tau| \rightarrow 0} A_{i}(\tau, \vec{x})=\tau^{\frac{1}{2}-p} U_{i}(\vec{x})
$$

And we consider the asymptotic symmetries of dilatations and inversion. The result is the same for both symmetries as in the scalar field, and then we only show the transformation law of the boundary field $U_{i}$ under dilatations.

Taking into account the transformation law for the vector field:

$$
A_{i}^{\prime}=\frac{\partial x^{j}}{\partial x^{\prime i}} A_{j}
$$

which for dilatations become $A_{i}^{\prime}=\lambda^{-1} A_{i}$. Then we can see that

$$
\begin{aligned}
A_{i}^{\prime} & =\tau^{\prime \frac{1}{2}-p} U_{i}^{\prime}\left(\overrightarrow{x^{\prime}}\right)=\lambda^{\frac{1}{2}-p} \tau^{\frac{1}{2}-p} U_{i}^{\prime}\left(\overrightarrow{x^{\prime}}\right) \\
& =\lambda^{-1} A_{i}=\lambda^{-1} \tau^{\frac{1}{2}-p} U_{i}(\vec{x}),
\end{aligned}
$$

so, $U_{i}$ transforms as

$$
U_{i}^{\prime}\left(\overrightarrow{x^{\prime}}\right)=\lambda^{-1-\left(\frac{1}{2}-p\right)} U_{i}(\vec{x})
$$

which, comparing with (20) implies that $U_{i}$ fulfil our expectations and behave precisely as a conformal vector field in $\mathbb{R}^{3}$ under the asymptotic symmetries of de Sitter group with conformal weight

$$
\Delta_{U}=1+\left(\frac{1}{2}-p\right)=\frac{3}{2}-p=\frac{3}{2}-\sqrt{\frac{1}{4}-\frac{m_{A}^{2}}{H^{2}}} .
$$

We learned in previous sections that in the spirit of the so called dS/CFT correspondence, free fields propagating on de Sitter admit a dual conformal field theory representations in the boundary of the space which we take to be a $\mathbb{R}^{3}$ space placed at the super horizon limit, this is at $|\tau| \ll|\vec{x}|$. We have calculated the conformal weight of the corresponding conformal fields correspondence which are essential for the calculation of correlation functions and to describe the statistical properties of the theory. Although the results presented in the previous sections where obtained for free fields, there are several discussions in the literature which points towards of an extension of this correspondence also in the presence of self interactions and for coupled 
systems involving several fields. In next section we discuss coupled system involving a scalar and a vector field which results useful for applications in inflationary cosmology and analyse in which conditions it is possible to extend the ideas explored in previous sections and if it is possible to find a conformal field theory representation of the theory aiming to calculate the properties of inflationary perturbations by using the conformal field theory machinery.

Vector-scalar coupled system. In this section we study a system of interacting scalar and vector fields which results useful for several applications to inflationary cosmology (Ratra, 1992; Dimopoulos et al., 2010a; Watanabe et al.,2010; Dimopoulos et al., 2010b; Wagstaff \& Dimopoulos, 2011; Barnaby et al., 2011; Sorbo, 2011; Dimopoulos \& Karciauskas, 2012; Anber \& Sorbo, 2012; Bartolo et al., 2013; Lyth \& Karciauskas, 2013; Shiraishi et al., 2013; Cook \& Sorbo, 2013; Nurmi \& Sloth, 2014; Caprini \& Sorbo, 2014; Chen et al., 2014; Bartolo et al., 2015; Namba et al., 2016). The model that we consider here is described by the action

$$
S_{\phi \mathrm{A}}=-\frac{1}{4} \int d^{4} x \sqrt{-g}\left[f_{1}(\phi) F^{\mu \nu} F_{\mu \nu}+f_{2}(\phi) \tilde{F}^{\mu \nu} F_{\mu \nu}\right] .
$$

In the second term, $\tilde{F}^{\mu \nu}$ is the Hodge dual of the field strength $F_{\mu \nu}$ and is defined by

$$
\tilde{F}^{\mu \nu}=\frac{1}{2 \sqrt{-g}} \epsilon^{\mu \nu \alpha \beta} F_{\alpha \beta},
$$

where $\epsilon^{\mu \nu \alpha \beta}$ is the four dimensional Levi-Civita symbol and $f_{1}(\phi)$ and $f_{2}(\phi)$ are coupling functions which depend only of the scalar field. This model introduces explicitly parity violation through the term $f_{2}(\phi) \tilde{F}^{\mu \nu} F_{\mu \nu}$. We assume the results obtained previously for the conformal weight of the scalar and the vector field in four dimensional de Sitter $\Delta_{\phi}=0$ and $\Delta_{A}=1$, so the action (48) is manifestly invariant under de Sitter group transformations. Additionally, assuming $\Delta_{\phi}=0$ imply that if we assign some conformal weight $\Delta_{f_{i}}$ to the coupling functions

$$
f_{i}^{\prime}\left(\phi^{\prime}\right)=\left|\operatorname{det}\left(\frac{\partial x^{\prime \mu}}{\partial x^{\nu}}\right)\right|^{-\frac{\Delta_{f_{i}}}{4}} f_{i}(\phi),
$$

we get that $\Delta_{f_{i}}=0$ in order to preserve de Sitter invariance. 
Now, we solve the equations of motion and study its super horizon evolution. In the context that we frame the discussion we consider that the scalar field drives the inflationary dynamics and that the vector field is an auxiliary subdominant field which affects the primordial curvature perturbation and can leave an imprint on the inflationary evolution. The scalar affect the evolution of the vector field through the coupling functions $f_{i}(\phi)$. The equations of motion for the vector field derived from the action (48) are:

$$
\nabla_{\mu}\left(f_{1}(\phi) F^{\mu \nu}+f_{2}(\phi) \tilde{F}^{\mu \nu}\right)=0
$$

which must be complemented with the Bianchi identity:

$$
\nabla_{\mu} \tilde{F}^{\mu \nu}=0
$$

We don't solve the dynamics of the scalar field, we just assume some features of its solutions due to the fact that this field drives the inflationary expansion. Accordingly, we further assume that the inflationary dynamics homogenise the scalar perturbations, so that we can approximate the scalar field as a time dependent function, this is $\partial_{i} \phi=0$. Accordingly, this implies that on the solutions of the inflationary scalar field $f_{1}(\phi)=f_{1}(\phi(\tau))$ and $f_{2}(\phi)=$ $f_{2}(\phi(\tau))$, then $\partial_{i} f_{1}=\partial_{i} f_{2}=0$. With this approximation, the temporal and spacial components of the equation (50) in de Sitter conformal coordinates (1) becomes respectively

$$
f_{1} \partial_{i} F_{i 0}=0
$$

and

$$
\left(\partial_{0} f_{1}\right) F_{0 i}-\left(\partial_{0} f_{2}\right) \varepsilon^{0 i j k} \partial_{j} A_{k}-f_{1}\left(\partial_{j} F_{j i}-\partial_{0} F_{0 i}\right)=0 .
$$

Given that this theory is manifestly gauge invariant because it only depend of the field strength $F_{\mu \nu}$ and its dual $\tilde{F}^{\mu \nu}$, we choose the Coulomb gauge and set $A_{0}=0$ and $\partial_{i} A_{i}=0$. With this choice, the temporal equation (52) cancels and the spatial part (53) reduces to:

$$
\left(\frac{\partial^{2}}{\partial \tau^{2}}-\nabla^{2}+\frac{1}{f_{1}} \frac{\partial f_{1}}{\partial \tau} \frac{\partial}{\partial \tau}+\frac{1}{f_{1}} \frac{\partial f_{2}}{\partial \tau} \nabla \times\right) \vec{A}(\tau, \vec{x})=0 .
$$

So far, we don't have any restriction over the form of the coupling functions $f_{i}$, and now we impose some conditions mainly, to preserve conformal invariance on the asymptotic region. Let's apply the dilatation $\tau^{\prime}=\lambda \tau$ and $\vec{x}^{\prime}=\lambda \vec{x}$ to the previous equation and demand invariance under this transformation. We further assume that the coupling functions are homogeneous functions of time, this is $f_{1}(\lambda \tau)=\lambda^{n} f_{1}(\tau), f_{2}(\lambda \tau)=\lambda^{m} f_{2}(\tau)$, so that the equation of motion transforms as: 


$$
\left(\frac{\partial^{2}}{\partial \tau^{2}}-\nabla^{2}+\frac{1}{f_{1}(\tau)} \frac{\partial f_{1}(\tau)}{\partial \tau} \frac{\partial}{\partial \tau}+\lambda^{m-n} \frac{1}{f_{1}(\tau)} \frac{\partial f_{2}(\tau)}{\partial \tau} \nabla \times\right) \vec{A}(\tau, \vec{x})=0
$$

In this way we see that a necessary condition for getting scaling invariance is that the coupling functions are homogeneous functions of the same order $n=m$. This is achieved if the couplings are power law functions proportional to each other:

$$
f_{1}(\tau)=c_{1}^{2}(-H \tau)^{n} \quad \text { and } \quad f_{2}(\tau)=c_{2}^{2}(-H \tau)^{n}
$$

which implies that

$$
f_{1}(\tau)=\gamma f_{2}(\tau) \quad \text { with } \quad \gamma=c_{2}^{2} / c_{1}^{2}
$$

In the previous expression, we restored the Hubble constant for dimensional analysis. Let us reuse $n=-2 \alpha$ because the function $f_{1}$ must be positive due to hamiltonian stability. In order to avoid strong coupling at super horizon evolution, we assume that $\alpha<0$

$$
f_{1}(\tau)=c_{1}^{2}(-H \tau)^{-2 \alpha}, f_{2}(\tau)=c_{2}^{2}(-H \tau)^{-2 \alpha} .
$$

Now, let's follow the analysis in terms of the normalized canonical field $a_{i}$ defined as

$$
A_{i}\left(\tau, x_{i}\right) \equiv \frac{a_{i}\left(\tau, x_{i}\right)}{\sqrt{f_{1}}} .
$$

For the canonical field $a_{i}$, the equation of motion (54) is written as

$$
\begin{array}{r}
\left\{\frac{\partial^{2}}{\partial \tau^{2}}-\nabla^{2}+\right. \\
+\frac{1}{2}\left[\frac{1}{2}\left(\frac{\partial_{\tau} f_{1}}{f_{1}}\right)^{2}-\frac{\partial_{\tau}^{2} f_{1}}{f_{1}}\right] \\
\left.+\frac{1}{f_{1}} \frac{\partial f_{2}}{\partial \tau} \nabla \times\right\} \vec{a}(\tau, \vec{x})=0
\end{array}
$$

and using (55) gives:

$$
\left(\frac{\partial^{2}}{\partial \tau^{2}}-\nabla^{2}-\frac{\alpha(\alpha+1)}{\tau^{2}}-\frac{2 \alpha \gamma}{\tau} \nabla \times\right) \vec{a}(\tau, \vec{x})=0
$$

Now, we go to Fourier space. Choosing $\vec{k}=(k, 0,0)$ and defining the transverse polarizations as

$$
a_{ \pm}=\frac{a_{y} \pm i a_{z}}{\sqrt{2}}
$$

we get

$$
\left(\frac{\partial^{2}}{\partial \tau^{2}}+k^{2}-\frac{\alpha(\alpha+1)}{\tau^{2}} \pm \frac{2 \alpha \gamma k}{\tau}\right) \tilde{a}_{ \pm}(\tau, \vec{k})=0
$$


The equation (59) can be solved analytically in terms of regular and irregular Whittaker functions $W$ and $M$ :

$$
\begin{aligned}
\tilde{a}_{ \pm}(\tau, \vec{k}) & =C_{1 \pm}(k) W_{\alpha+1 / 2}(-i \xi, 2 i k \tau) \\
& +C_{2 \pm}(k) M_{\alpha+1 / 2}(-i \xi, 2 i k \tau)
\end{aligned}
$$

where the parameter $\xi=-\alpha \gamma$ defined in Caprini \& Sorbo (2014) determine the relative size of the parity violation signals in the model. Reference Caprini \& Sorbo (2014) study in full detail the features of the model with $f_{1}=\gamma f_{2}$ and describe analytically its asymptotic behaviour. For scales in which $|k \tau| \ll \xi$, the $W$ function dominates and we will consider only its contribution. Morover, if we take $|k \tau| \ll \xi$ in (59) we have solutions in terms of modified Bessel functions of the first kind $\mathcal{I}_{\nu}$ :

$$
\begin{aligned}
\tilde{a}_{ \pm}(\tau, \vec{k}) \approx & C_{1 \pm}(k) \sqrt{-2 \xi k \tau} \mathcal{I}_{-(1+2 \alpha)}(\sqrt{-8 \xi k \tau}) \\
& +C_{2 \pm}(k) \sqrt{-2 \xi k \tau} \mathcal{I}_{(1+2 \alpha)}(\sqrt{-8 \xi k \tau})
\end{aligned}
$$

Which, in particular, for super horizon scales in which $|8 \xi k \tau| \ll 1$ we have

$$
\begin{aligned}
\tilde{a}_{ \pm}(\tau, \vec{k}) \approx & {\left[C_{1 \pm}(k)(-2 \xi k \tau)^{\alpha+1} \Gamma(-1-2 \alpha)\right.} \\
& \left.+C_{2 \pm}(k)(-2 \xi k \tau)^{-\alpha} \Gamma(1+2 \alpha)\right] .
\end{aligned}
$$

We can write the last equation as

$$
\tilde{a}_{ \pm}(\tau, \vec{k}) \approx \tilde{u}_{ \pm}(k)(-\xi H \tau)^{\alpha+1}+\tilde{v}_{ \pm}(k)(-\xi H \tau)^{-\alpha},
$$

in which we restored $H$ and absorbed further dependence of $k, \xi, \alpha$ in the $\tilde{u}$ and $\tilde{v}$ functions. At this point, we go back to coordinates space obtaining the asymptotic form:

$$
a_{ \pm}(\tau, \vec{x}) \approx u_{ \pm}(\vec{x})(-\xi H \tau)^{\alpha+1}+v_{ \pm}(\vec{x})(-\xi H \tau)^{-\alpha} .
$$

We have succeed on separating the time and space coordinates for super horizon scales, so, we can assign a conformal boundary field in this case as in the previous cases studied. This situation is nevertheless a bit different given that the dominant term depends on the value of the exponent $\alpha$, if $\alpha>-1 / 2$ the term $v_{ \pm}$dominates, while, if $\alpha<-1 / 2$ the term $u_{ \pm}$is the one that dominates. If $\alpha>-1 / 2$, we demand that

$$
\lim _{|\tau| \rightarrow 0} a_{ \pm}(\tau, \vec{x})=\tau^{-\alpha} v_{ \pm}(\vec{x})
$$

while, if $\alpha<-1 / 2$ we take the boundary condition

$$
\lim _{|\tau| \rightarrow 0} a_{ \pm}(\tau, \vec{x})=\tau^{\alpha+1} u_{ \pm}(\vec{x}) .
$$


Now, we can calculate the conformal weight of those boundary fields applying the same procedure of previous sections. We only consider the dilatation transformation, the inversion is entirely analogous. We see that the canonical field $a_{ \pm}$transforms under four dimensional dilatations $\tau^{\prime}=\lambda \tau, x^{\prime}=\lambda x$, $x_{ \pm}^{\prime}=\lambda x_{ \pm}$as a four dimensional vector with weight $\Delta_{a}=1$ :

$$
a_{ \pm}^{\prime}=\frac{\partial x_{ \pm}}{\partial x_{ \pm}^{\prime}} a_{ \pm}=\lambda^{-\Delta_{a}} a_{ \pm}=\lambda^{-1} a_{ \pm} .
$$

Applying dilatation over (64) we have:

$$
\begin{aligned}
a_{ \pm}^{\prime} & =\left(\tau^{\prime}\right)^{-\alpha} v_{ \pm}^{\prime}\left(\vec{x}^{\prime}\right)=\lambda^{-\alpha}(\tau)^{-\alpha} v_{ \pm}^{\prime}\left(\vec{x}^{\prime}\right) \\
& =\lambda^{-1} a_{ \pm}=\lambda^{-1}(\tau)^{-\alpha} v_{ \pm}(\vec{x})
\end{aligned}
$$

which implies

$$
v_{ \pm}^{\prime}\left(\vec{x}^{\prime}\right)=\lambda^{\alpha-1} v_{ \pm}(\vec{x})
$$

and comparing with (20) with $n=1$ and $d=3$ we conclude that the boundary field $v_{ \pm}$behave as a conformal field of weight:

$$
\Delta_{v}=1-\alpha .
$$

Following the same procedure we find that the boundary field (65) behaves as a conformal boundary field of weight

$$
\Delta_{u}=\alpha+2
$$

To summarise, we have obtained that the interacting system (48) admit a conformal field theory representation at super horizon scales through the boundary vector fields $u_{ \pm}, v_{ \pm}$. A remarkable fact of this results is that they coincide with the case studied in Biagetti et al. (2013), where they studied the model without parity violation term, this is

$$
S_{\phi \mathrm{A}}=-\frac{1}{4} \int d^{4} x \sqrt{-g} f_{1}(\phi) F^{\mu \nu} F_{\mu \nu}
$$

Naively, we could think that both cases, the parity conserving and the parity violating models have the same conformal boundary field associated and that the statistical features of the theories given by the correlating functions are the same. Nevertheless, there is a crucial difference implied by the fact that the super horizon boundary symmetry group allow the spacial reflection $\vec{x}^{\prime}=-\vec{x}$ as an element of the symmetry group. In this case, the correlating functions allow for antisymmetric terms in its structure, for instance, the power spectrum of a boundary field would be

$$
\left\langle u_{i}(\vec{k}) u_{j}(-\vec{k})\right\rangle=\left(\beta_{1}(k) \delta_{i j}-\beta_{2}(k) \hat{k}_{i} \hat{k}_{j}+\beta_{3}(k) \epsilon_{i j a} k_{a}\right)
$$


where the form of the $\beta_{i}$ functions can be determined by using the asymptotic symmetries of the theory (Biagetti et al., 2013). It is important to see that the functions $\beta_{2}(k)$ and $\beta_{3}(k)$ are related to anisotropic and to parity violating effects respectively. In the parity conserving case $\left(\beta_{3}=0\right)$, the above equation can be written as

$$
\left\langle u_{i}(\vec{k}) u_{j}(-\vec{k})\right\rangle=\beta_{1}(k)\left(\delta_{i j}-\hat{k}_{i} \hat{k}_{j}\right)
$$

If we assume that the vector field points along the direction of some unit vector $\hat{\mathbf{n}}$ and the wave vector $\mathbf{k}$ points along the unit vector $\hat{\mathbf{k}}$, the power spectrum can be written as (Ackerman et al., 2007):

$$
\beta(\mathbf{k})=\beta(k)\left(1+g(k)(\hat{k} \cdot \hat{n})^{2}\right),
$$

where $\beta(k)$ is the isotropic power spectrum and $g(k)$ is a function measuring the amount of statistical anisotropy. If $g(k)$ is scale invariant, recent data analysis gives an upper bound on that: $g \leq 10^{-2}$ (Kim \& Komatsu, 2013; Ade et al., 2016).

A further development of this ideas on the calculation of the correlation functions using conformal field theory techniques exploiting the asymptotic symmetries as presented in Biagetti et al. (2013) for the case of parity violating theories have enough interests and we expect to pursue this possibility in future work.

\section{Conclusions}

In this paper, we have comprehensively studied the symmetry properties of the scalar and vector fields solutions on de Sitter space. We study their asymptotic limit at super horizon scales and we found that, in this limit, free fields behave as conformal fields on a constant time surface. We study the free scalar and the free vector fields for pedagogical purposes, to illustrate the technique and then study an inflationary model with interacting scalar and vector fields.

The coupled system is studied because it is interesting for its cosmological implications, in particular this kind of model can explain some observable CMB anomalies such as broken of inversion and rotational invariance. The super horizon scales are important in our context because inflationary expansion freeze the perturbations at this scales and the correlation functions, evaluated at this scales, carry information from the inflationary dynamics. In this sense, the use of the asymptotic conformal symmetries of de Sitter space offers a powerful technique to uncover the structure of the correlations, which relies only of the conformal weight of the boundary asymptotic fields. 
Regarding the specific scalar-vector model studied, we can mention two important aspects, the first one is that the coupling functions $f_{1}(\phi)$ and $f_{2}(\phi)$ in Eq. (48) fulfill the condition $f_{2}(\phi) / f_{1}(\phi)=$ constant and the second one is that if we assume that the functions are a power law on $H \tau$ (which is a good choice because we are looking for an homogeneous universe), the conformal weight of the vector fields on super horizon scales depend only of that power (see Eqs. (69) and (70)). This result is according with the one obtained by Biagetti et al. (2013), who studied the parity conserving case with $f_{2}(\phi)=0$. Our next step is to use the conformal weight of the boundary vector field to calculate the shape of the correlation functions following the procedure given by Biagetti et al. (2013) and identify the signals of parity violation.

\section{Acknowledgements}

This work was partially supported by COLCIENCIAS grants numbers 11065693 3958 RC 0384-2013 and 123365843539 RC FP44842-081-2014 and by COLCIEN CIAS-ECOS NORD grant number RC 0899-2012. C.A.V.-T. acknowledges financial support from Vicerrectoría de Investigaciones (Univalle) grant number 7924. JPBA thanks Universidad del Valle for its warm hospitality during several stages of this project.

\section{Conflict of interest}

None.

\section{References}

Ackerman L, Sean C, Mark W. Imprints of a Primordial Preferred Direction on the Microwave Background, Physical Review D, 75: 083502, 2007. doi: 10.1103/PhysRevD.75.083502

Ade PAR, et al. Planck 2015 results. Isotropy and statistics of the CMB, Astronomy \& Astrophysics, 594(A16): 1-62, 2016.

doi: $10.1051 / 0004-6361 / 201526681$

Anber M, Sorbo L. Non-Gaussianities and Chiral Gravitational Waves in Natural Steep Inflation, Physical Review D, 85: 123537, 2012.

doi: 10.1103/PhysRevD.85.123537

Barnaby N, Namba R, Peloso M. Phenomenology of a Pseudo-Scalar Inflaton: Naturally Large Nongaussianity, fournal of Cosmology And Astroparticle Physics, 1104: 009. 2011.

doi: 10.1088/1475-7516/2011/04/009 
Bartolo N, Matarrese S, Peloso M, Ricciardone A. The anisotropic Power Spectrum and bispectrum in the $\mathrm{f}($ )F2 Mechanism, Physical Review D, 87: 023504, 2013.

doi: 10.1103/PhysRevD.87.023504

Bartolo N, Matarrese S, Peloso M, Shiraishi M. Parity-violating CMB Correlators With Non-decaying Statistical Anisotropy, fournal of Cosmology and Astroparticle Physics, 07: 039, 2015.

doi: 10.1088/1475-7516/2015/07/039

Biagetti M, Kehagias A, Morgante E, Perrier H, Riotto A. Symmetries of Vector Perturbations During the de Sitter Epoch, fournal of Cosmology and Astroparticle Physics, 1307030, 2013.

doi: 10.1088/1475-7516/2013/07/030

Bousso R, Maloney A, Strominger A. Conformal Vacua and Entropy in de Sitter space, Physical Review D, 65: 104039, 2002.

doi: 10.1103/PhysRevD.65.104039

Caprini Ch, Sorbo L. Adding Helicity to Inflationary Magnetogenesis, fournal of Cosmology and Astroparticle Physics, 1410056, 2014.

doi: 10.1088/1475-7516/2014/10/056

Chen X, Emami R, Firouzjahi H, Wang Y. The TT, TB, EB and BB Correlations in Anisotropic Inflation, fournal of Cosmology and Astroparticle Physics, 1408027, 2014.

doi: $10.1088 / 1475-7516 / 2014 / 08 / 027$

Cook JL, Sorbo L. An Inflationary Model With Small Scalar and Large Tensor Nongaussianities, Journal of Cosmology and Astroparticle Physics, 1311047, 2013.

doi: $10.1088 / 1475-7516 / 2013 / 11 / 047$

Dimastrogiovanni E, Bartolo N, Matarrese S, Riotto A. Non-Gaussianity and Statistical Anisotropy from Vector Field Populated Inflationary Models, Advances in Astronomy, 752670, 2010.

doi: $10.1155 / 2010 / 752670$

Dimopoulos K, Karciauskas M. Parity Violating Statistical Anisotropy, fournal of High Energy Physics, 06040, 2012.

doi: 10.1007/JHEP06(2012)040 
Dimopoulos K, Karciauskas M, Lyth DH, Rodriguez Y. Statistical Anisotropy of the Curvature Perturbation from Vector Field Perturbations, fournal of Cosmology and Astroparticle Physics, 0905013, 2009.

doi: 10.1088/1475-7516/2009/05/013

Dimopoulos K, Karciauskas M, Wagstaff JM. Vector Curvaton With Varying Kinetic Function, Physical Review D, 81023522. 2010a.

doi: 10.1103/PhysRevD.81.023522

Dimopoulos K, Karciauskas M, Wagstaff JM. Vector Curvaton without Instabilities, Physics Letters B, 683 (4-5): 298 -301, 2010b

doi: 10.1016/j.physletb.2009.12.024

Ginsparg P. Applied Conformal Field Theory. In Les Houches Summer School in Theoretical Physics: Fields, Strings, Critical Phenomena Les Houches, France, June 28-August 5, 1988.

Golovnev A, Viatcheslav M, Vitaly V. Vector Inflation, fournal of Cosmology and Astroparticle Physics, 0806009. 2008.

doi: 10.1088/1475-7516/2008/06/009

Gumrukcuoglu AE, Himmetoglu B, Peloso M. Scalar-Scalar, Scalar-Tensor, and Tensor-Tensor Correlators from Anisotropic Inflation, Physics Review D, 81063528, 2010.

doi: 10.1103/PhysRevD.81.063528

Himmetoglu B, Contaldi CR, Peloso M. Instability of Anisotropic Cosmological Solutions Supported By Vector Fields, Physical Review Letters, 102111301. 2009a.

doi: 10.1103/PhysRevLett.102.111301

Himmetoglu B, Contaldi CR, Peloso M. Instability of the ACW Model, and Problems With Massive Vectors During Inflation, Physical Review D, 79063517. 2009b.

doi: 10.1103/PhysRevLett.102.111301

Kim J, Komatsu E. Limits on anisotropic inflation from the Planck data, Physics Review D, 88: 101301, 2013.

doi: 10.1103/PhysRevD.88.101301 
Leblond F, Marolf D, Myers RC. Tall Tales from de Sitter Space 1: Renormalization Group Flows, Journal of High Energy Physics, 06052. 2002.

doi: 10.1088/1126-6708/2002/06/052

Leblond F, Marolf D, Myers RC. Tall Tales from de Sitter Space. 2. Field Theory Dualities, fournal of High Energy Physics, 01003. 2003.

doi: 10.1088/1126-6708/2003/01/003

Lyth $\mathrm{DH}$, Karciauskas M. The statistically anisotropic curvature perturbation generated by $f^{2}(\phi) F_{\mu \nu} F^{\mu \nu}$, fournal of Cosmology and Astroparticle Physics, 1305, 011, 2013.

doi: $10.1088 / 1475-7516 / 2013 / 05 / 011$

Lyth DH, Malik KA, Sasaki M. A General Proof of the Conservation of the Curvature Perturbation, Journal of Cosmology and Astroparticle Physics, 0505, 004. 2005.

doi: 10.1088/1475-7516/2005/05/004

Maleknejad A, Sheikh-Jabbari MM, Soda J. Gauge Fields and Inflation, Physics Reports, 528:161 - 261, 2013.

doi: $10.1016 /$ j.physrep.2013.03.003

Nakamura T, Stewart ED. The Spectrum of Cosmological Perturbations

Produced by a Multicomponent Inflaton to Second Order in the Slow Roll Approximation, Physics Letters B, 381: 413-419, 1996.

doi: 10.1016/0370-2693(96)00594-1

Namba R, Peloso M, Shiraishi M, Sorbo L, Unal C. Scale-dependent gravitational waves from a rolling axion, Journal of Cosmology and Astroparticle Physics, 1601041, 2016.

doi: 10.1088/1475-7516/2016/01/041

Nurmi S, Sloth MS. Constraints on Gauge Field Production during Inflation, Journal of Cosmology and Astroparticle Physics, 1407012, 2014.

doi: 10.1088/1475-7516/2014/07/012

Ratra B. Cosmological 'Seed' Magnetic Field from Inflation, Astrophysical fournal, 391(1): L1-L4, 1992.

doi: $10.1086 / 186384$ 
Sasaki M, Stewart ED. A general analytic formula for the spectral index of the density perturbations produced during inflation, Progress of Theorical Physics, 95(1):71-78, 1996.

doi: 10.1143/PTP.95.71

Shiraishi M, Ricciardone A, Saga S. Parity violation in the CMB bispectrum by a rolling pseudoscalar, fournal of Cosmology and Astroparticle Physics, 1311051, 2013.

doi: $10.1088 / 1475-7516 / 2013 / 11 / 051$

Soda J. Statistical Anisotropy from Anisotropic Inflation, Classical and Quantum Gravity, 29(8):083001, 2012.

doi: $10.1088 / 0264-9381 / 29 / 8 / 083001$

Sorbo L. Parity violation in the Cosmic Microwave Background from a pseudoscalar inflaton, Journal of Cosmology and Astroparticle Physics, 1106003, 2011.

doi: $10.1088 / 1475-7516 / 2011 / 06 / 003$

Spradlin M, Strominger A, Volovich A. Les Houches lectures on de Sitter space. In Unity from duality: Gravity, gauge theory and strings. Proceedings, NATO Advanced Study Institute, Euro Summer School, 76th session, Les Houches, France, 423-453, 2001.

Spradlin M, Volovich A. Vacuum states and the S matrix in dS \CFT, Physical Review D, 65:104037, 2002.

doi: 10.1103/PhysRevD.65.104037

Starobinsky AA. Multicomponent de Sitter (Inflationary) Stages and the Generation of Perturbations, Journal of Experimental and Theoretical Physics Letters, 42, 152-155, 1985.

Strominger A. The dS $\backslash$ CFT correspondence, Journal of High Energy Physics, 10034, 2001.

doi: $10.1088 / 1126-6708 / 2001 / 10 / 034$

Tanhayi MR. Quasinormal modes in de sitter space: Plane wave method, Physical Review D, 90, 064010, 2014.

doi: 10.1103/PhysRevD.90.064010 


\section{Wagstaff JM, Dimopoulos K. Particle Production of Vector Fields: Scale \\ Invariance is Attractive, Physical Review D, 83:023523, 2011. \\ doi: 10.1103/PhysRevD.83.023523}

\section{Watanabe M, Kanno S, Soda J. The Nature of Primordial Fluctuations from Anisotropic Inflation, Progress of Theoretical Physics, 123: 1041-1068, 2010. doi: 10.1143/PTP.123.1041}

Simetrías en el espacio de De Sitter y modelos inflacionarios escalar-vector

Resumen. En este artículo se estudia la correspondencia entre una teoría de campos en el espacio de De Sitter en $D$-dimensiones y una teoría dual conforme en un espacio euclidiano en $(D-1)$-dimensiones. En particular, se investiga la forma en la que se establece esta correspondencia para un sistema compuesto por un campo escalar y un campo vectorial acoplados propagándose en el espacio de De Sitter. Se analizan algunas condiciones necesarias (pero no suficientes) en las cuales la simetría conforme se preserva en la teoría dual en $(D-1)$-dimensiones y hace posible el establecimiento de la correspondencia. La discusión presentada aquí se enmarca en el contexto de la cosmología inflacionaria, así que los resultados obtenidos plantean algunas posibilidades relevantes de aplicación en el cálculo de funciones de correlación de los campos y de la perturbación de la curvatura primordial $\zeta$ en modelos inflacionarios que incluyen campos escalares y vectoriales acoplados.

Palabras clave: inflación; campos vectoriales; simetrías de De Sitter; violación de paridad.

Simetrias de De Sitter e modelos inflacionarios escalar-vetors

Resumen. Neste artigo, é estudada a correspondência entre uma teoria de campos no espaço de De Sitter em $D$-dimensões e uma teoria dual conforme num espaço euclidiano em $(D-1)$-dimensões. Em particular, é pesquisado o caminho no qual se estabelece esta correspondência para um sistema de interação composto dum campo escalar e um vetorial, propagando-se no espaço de De Sitter. São analisadas algumas condições necessárias (mas não suficientes) para as quais a simetria conforme é preservada na teoria dual em (D-1)-dimensões, fazendo viável o estabelecimento da correspondência. A discussão apresentada aqui, está situada no contexto da cosmologia inflacionária. Portanto, os resultados obtidos representam algumas possibilidades relevantes para sua aplicacão no cálculo de funções de correlação dos campos e da perturbação da curvatura primordial $\zeta$ em modelos inflacionários incluindo campos acoplados escalares e vetoriais.

Palabras clave: inflação; campos vetoriais; simetrias de de Sitter; violação da paridade. 
Josue Motoa Manzano

Is currently MsC student at Universidad del Valle where he earned his BSc in physics. His research area is theoretical cosmology, and his main current research interest is inflationary physics.

\section{Juan P. Beltrán Almeida}

Is assistant professor at Universidad Antonio Nariño since 2010. The author coursed undergraduate studies at Universidad del Valle and obtained his $\mathrm{PhD}$ in physics at the Instituto de Física Teórica at Universidade Estadual Paulista (IFT-UNESP) in São Paulo. His research area is theoretical cosmology, and his main research interests are inflationary physics and the large scale structure formation in the Universe.

\section{César A. Valenzuela-Toledo}

Is assistant professor at Universidad del Valle since 2010. The author earned his $\mathrm{PhD}, \mathrm{MsC}$ and BSc in physics from Universidad Industrial de Santander. He is a theoretical cosmologist working on the properties of the early Universe. His main research interest is to study the physics of inflation and the large scale structure formation in the Universe. 